\title{
The Design and Implementation of Florist's Shop Online System Based on J2EE
}

\author{
Hui Shi \\ Department of Information Engineering, Henan Polytechnic \\ Zhengzhou, Henan, China 450046
}

\author{
Hui Zhai \\ Department of Information Engineering, Henan Polytechnic \\ Zhengzhou, Henan, China 450046 \\ e-mail: hnzh1121@163.com
}

\author{
Rui Zhai \\ South No.1 community, Yungang streets, Fengtai \\ Beijing, China 100074 \\ e-mail: zk_rui@163.com
}

\begin{abstract}
With the rapid development of Internet, e-commerce had been widely using by more and more people, so had shopping online. The J2EE architecture was adopted in this paper. An florist's shop online system was designed and implemented based on the analysis of the actual needs of users. Through running test for a period of time, the system could be operated steadily, and its function was complete. The system could achieve flower sales on line that was highly efficient and convenient, and the design purpose was achieved also.
\end{abstract}

Keywords- e-commerce; florist's shop online system; J2EE; web application

\section{INTRODUCTION}

With the rapid development of the Internet since 2000, the e-commerce market in China presented steady growth with $40-50 \%$ of the high speed in ten years. The electronic commerce form came into thousands of households, and became an important part of life between the enterprise and the consumer. ${ }^{[1]}$ At present, the main character of electronic commerce market in our country was the prevalence of C2C(Consumer to Consumer) transaction. A lot of C2C transactions were on Taobao in Alibaba Group Holding Ltd. ${ }^{[2]}$ More and more people were using shopping online which was new pattern of consumption now.And the business activities by using network which was electronic means had been adopted quickly by the majority of businessmen. Nowadays, a huge florist's industry group had formed in our country with the improvement of living standards. As the important link in the flower sales system, the florist's couldn't meet the needs of the vast number of consumers yet after the traditional sales mode just like flowers retail, flower art design etc. To development of an online flower shop sales system had become the urgent need to resolve problems.

\section{J2EE TECHNOLOGY OVERVIEW}

At present, Java was the most widely used in electronic commerce system development technology. Because its reuse degree was strong, and it could support more development tools and cross platform also. In constructing the florist's shop online system, the use of J2EE technology were mainly JSP, EJB, JDBC, etc. Among them, the JSP (Java Server Pages) was a kind of dynamic web technology standard. ${ }^{[3]}$ EJB (Enterprise JavaBean)was a part of J2EE, which defined a standards based on component enterprise to develop multiple application. ${ }^{[4]}$ It provided a distributed logic framework about development and implementation, which simplified application development largely of enterprise. JDBC (Java Data Base Connectivity) was a kind of Java API to be used to execute SQL statement. It could provide a unified access for a variety of relational database. It was consisted of a set of class and interface composition byJava language writing. ${ }^{[5]}$ J2EE ( Java 2 Platform,Enterprise Edition) was a system structure related to the complicated problem of development, deployment and management,which simplified enterprise solutions by using of Java 2 platform. ${ }^{[6]}$ The florist's shop online system was using the J2EE architecture.

III.

\section{SYSTEM DESIGN AND IMPLEMENTATION}

\section{A.Analysis on System Function}

After the analysis of the demand of the florist's shop online system, we could come to the system including two parts: the foregrounding and background management .The main function was achieved by front desk operation as follows:

(1)Registered function: to provide user registration function. The user could be a member of the flower shop online through the registration.

(2)Personal information management functions: the user could modify the personal data submitted, inquire the order and the consumption on the web site in condition of having registered cases.

(3)Shopping function: the user could browse flower product information after login, or search flower product that's he needed according to the query conditions also, then choose and buy flower product, and delivery order and checkout on-line through shopping cart and the cashier's function in this website.

(4)Evaluation function: the users could evaluate the transaction after shopping success.

(5)Cancellation function: the cancellation function must 
be provided for the user to quit the system safely of withdrawal from the system.

Background management was the management of this web site. The system administrator was exist in the initialization of system. They were added into the database manually in the database design. The main functions were realized as follows:

(1)Flower product management: to add, delete and show of classification about flower product .And it can be used recommend flower product to users based on flower product sales and each manufacturer prestige.

(2)User management:to management member information and administrator information in web site.

(3)Announcement Settings: to manage announcement information on web site. The announcement on web site could be added, deleted and modified.

(4)Order management: to handle the orders submitted by user timely in time

(5)Link management: to set website links. The website links could be added, deleted and modified.

(6)Cancellation function: the cancellation function must be provided when the management exited system, and it must be safe to withdrawal from the system.

\section{B.System Database Design}

After analyzing of the system function, the list was used mainly as follows:

(1) Admin(LoginId、LoginPwd);

(2)User(UserId 、UserName 、UserPwd 、 UserSex 、 UserTel、UserAdd、UserEmail);

(3) Flower(FlowerID 、FlowerName 、FlowerSort 、 FlowerOrigin、FlowerNumber、FlowerColor、FlowerPrice、 FlowerDescribe);

(4) FlowerCategory (FCId、FCName);

(5)Notice(NoticeId、 NoticeDescribe);

(6)OrderForm(OFId 、FlowerId 、FlowerNumber 、 OFTime、OFModeOfPayment、OFIsship

、OFTypeOfPayment、OFIspay、OFTotalMoney);

(7)Linker(LinkerId、LinkerDescribe);

\section{Implementation of the System}

There're much more functions realized by the system is , but the key algorithm of some functions could be given only limited to this paper length.

1. Public class design

(1) Acquisition system time class

Counting system time class was CountTime, the system current time could be obtained by currentlyTime() methods for in the operation of the class. The specific algorithm was as follows:

Define method currentlyTime(),return value is string: Date date $=$ new Date () ;

$$
\text { DateFormat dateFormat }
$$

DateFormat.getDateInstance(DateFormat.FULL); return dateFormat.format(date);

(2) Database connection class

Created database connection class JBDConnection, set the driver of connection SQL Server 2000 , the address of url and the instance of declaration connection class in the attribute. Then connected to database by using structure method.

2.The realization of the method checkMember() to verify user's identity of the user login function

When the user was online shopping in this system, the identity authentication must be conduct in order to ensure the safety of data information. When the user input the user name and password, it would call the method checkMember() to verify user identity.The specific algorithm was as follows:

define strings called name= request.getParameter(“name”); define

MemberForm memberForm=dao.selectMemberForm(name);

if( memberForm equal null) \{reauest.setAttribute("resutlt”, “The user does not exist.please enter again!”);\}

else

if(!memberForm.getPassword().equals(request.getParameter (“password").trim()))

\{request.setAttribute("result", "Password is wrong,please enter again!”)\} \} ;

else\{request.setAttribute(“memberForm”,memberForm)

return mapping.findForward(“checkMember”);

The user login results would be displayed in $\mathrm{f}$ checkMemberResult after implementation of user validation. JSP page was in f-checkMemberResult.jsp page, it would got the user's login result by request's object getAttribute().If the user login validation was successful, it would return to the home page of the florist's shop online system directly. If not, the results would be shown by JavaScript script and return to your home page.

3.The realization of the function of the shopping cart

The consumers would put the goods which had be chosen already into the shopping cart (basket) in the supermarket shopping, and bought them in the cashier's check after the completion of the choose. In the online shopping process, the "shopping cart" technology was adopted usually to simulate the real life shopping cart (basket). In the florist's shop online system, it could not only to add, view, modify, empty the flower product had been chosen at any time through the shopping cart, but also could buy them to the cashier's check at any time. The front desk shopping cart module mainly included adding flower product to shopping cart, querying flower product in shopping car, modifying the quantity of flower product in shopping cart, removing the flower products in shopping cart and emptying shopping cart, etc.

(1)The realization of adding flower product to the shopping cart

The users could view the flower product detailed information while opening a flower product button hyperlink in the home page. When he clicked the "put the shopping cart" button, then the cart_add.jsp page would be triggered. The page would store product information temporarily in the shopping cart. The object cart of Vector 
type was adopted to store flower product data in shopping cart and was saved in the client session object. When the flower product was added to the shopping cart, there would be two situations:

The first one was when the cart was empty, that was to say, when the user add the first flower product into the shopping cart, a cart must be created, then the flower product information could be saved to the cart variables.

The second case was when the cart was not empty, that was to say, some flower products had been chosen already in the shopping cart. Then the user could add the flower products into the shopping cart directly. If the flower product was reduplicate, the quantity of the flower products in cart needed to be modified. Specific algorithm was as follows:

The value of goodsID is Interger.parseInt(request.getParameter(goodsId));

The value of goodsPric

Float.parseFloat(request.getParameter(“price”));

Define sellGoodForm=new SellGoodsForm();

The value of sellGoodForm.ID isgoodsID;

The value of sellGoodForm.price is goodsPrice;

The value of sellGoodForm.number is 1 ;

The value of flag is true;

The value of

(Vector)session.getAttribute(“cart”);

if(cart equal null) cart= new Vector()

else $\{$ for $($ int $\mathrm{i}=0 ; \mathrm{i}<$ cart.size ()$; \mathrm{i}++)\{$

SellGoodsForm

form=(SellGoodsForm)cart.elementAt(i);

if(form.ID

equal

sellGoodsForm.ID)\{Form.number++;Cart.setElementAt(for

m,i);Flag=false $\}\}$;

(2)The realization of the empty shopping cart

Emptied all purchase information of the flower product stored in the session:session.removeAttribute("cart"); The page was returned to the flower cart was empty : response.sendRedirect(“cart-see.jsp”);

\section{CONCLUSION}

The florist's shop online system was an important application of e-commerce sales model. It could realize online trading of flowers. In the rapid development of the Internet today, the florist's shop online system would have a great market potential because the flowers had certain refreshing time. Based on elaborating the necessity of development of the florist's shop online system, the J2EE technology to develop the system was introduced at first in this paper. then the user demand of the system was analysed deeply. A scientific and reasonable database was designed according to the customer demand. Finally, the design of the whole system and the key code of implementation part in the system realization was given. After a period of time in the system test and trial operation, it had been shown that the system had accomplished the desired basic goal. And it had a certain inspiration and reference significance to other similar online shopping system. However, it was a complicated project to develop the florist's shop online system, the further improvement of system must be done combined the problems appeared in the course of the actual use.

\section{REFERENCES}

[1] Huangxianying. The design of servlet in the J2EE platform. Computer application research 2002.01:140-141

[2] Duming,Lichaochun. the design and implementation of Struts+EJB in J2EE platform [J], Computer application research,2005(3):223-225,228

[3] Feisike product research and development center.JSP application development steps.Beijing:Publishing house of electronics industry,2002

[4] http://baike.baidu.com/view/3542.htm

[5] http://baike.baidu.com/view/25611.htm

[6] http://baike.baidu.com/view/4242783.htm 